\title{
The Relationship between Environmental Factors and the Onsets of Avian Influenza Outbreaks in Thailand
}

\author{
Hirun Sawaengkaew \\ Environmental Science (Interdisciplinary Program) \\ Graduate School, Chulalongkorn University \\ Phyathai Road, Pathumwan, Bangkok 10330, Thailand \\ E-mail: hiruns@yahoo.com
}

Surat Baulert (Corresponding author)

Department of General Science, Faculty of Science, Chulalongkorn University

Phyathai Road, Pathumwan, Bangkok 10330, Thailand

E-mail: surat.b@chula.ac.th

Kumthorn Thirakhupt

Department of Biology, Faculty of Science, Chulalongkorn University

Phyathai Road, Pathumwan, Bangkok 10330, Thailand

E-mail: Kumthorn.T@chula.ac.th

This work was funded by The 90 ${ }^{\text {th }}$ Anniversary of Chulalongkorn University Fund (2008)

(Ratchadaphiseksomphot Endowment Fund)

\begin{abstract}
The relationship between environmental factors and the onsets of avian influenza virus (AIV; H5N1) outbreaks in Thailand was studied. The results show that the onsets of the disease correlate with the maximum gradient of temperature and wind speed within 24 hours, density of villages in a radius of five kilometers, and distance from the last AIV outbreak area. This study also presents how meteorological conditions have an effect on the occurrence of onsets of AIV in Thailand.
\end{abstract}

Keywords: Environmental factors, Avian influenza

\section{Introduction}

Avian influenza virus (AIV) or bird flu is one of the avian diseases. It can be classified in the Family Orthomyxoviridae and has four types: A, B, C, and D (Thogotovirus). These four types can be further subdivided by Glycoproteins, Hemagglutinin $(\mathrm{H})$ and Neuraminidase $(\mathrm{N})$, which have 16 and 9 subtypes, respectively (Fouchier et al., 2005). AIV is a highly pathogenic avian influenza, which is classified as type A, H5N1. According to the first report of an AIV outbreak in 1997 on Hong Kong Island, it caused an infection in poultry and wild birds, leading to a great number of deaths, and a rapid spread to many countries consequently. Although the disease affected birds, other studies also showed that the infection had caused the death of other animals and human. At present, 262 patients have died from this disease (World Health Organization, 2009).

In Thailand, AIV was first reported in poultry and wild birds in 2004. From 2004 - 2006, the deaths of 17 patients were reported because of infection by AIV. The government established defensive measures to control or to inhibit the spread of AIV by destroying poultry in many areas (Bureau of Disease Control and Veterinary Services, 2006), but the epidemic recurred in these areas. It was thought that there might be other factors involved in the outbreak. Water bird migration, for instance, was presumed to be a factor in the outbreak because the water birds survived AIV (Webster et al., 1992). That meant that if the pandemic occurred along with the migration of water birds, they would have the infection and become carriers to other areas (Sturm-Ramirez et al., 2005; Zhou et al., 2006). However, if the pandemic does not match just the range of bird migration, environmental factors would be seen to assist in spreading the disease to other areas. 
At present, scientific reports have found relationships between AIV and several limiting factors namely temperature, $\mathrm{pH}$, and medicinal liquids (World Health Organization, 2009) as well as the ability of AIV to survive in water for 3 days (Songserm et al., 2005). Nevertheless, none of the literature has mentioned the relationship of environmental factors to the AIV outbreak and with the onsets of AIV outbreaks. Therefore, this paper describes the relationship between environmental factors and the onsets of the AIV outbreaks in Thailand, in order to plan disease control in the future.

\section{Materials and Methods}

\subsection{Data preparation}

2.1.1 Data about the onsets of AIV outbreaks in Thailand from 2004 to 2008 was taken from the reports by The World Organization for Animal Health (OIE: Office International des Epizooties) and the Department of Livestock Development Thailand (DLD).

2.1.2 Data of meteorology in Thailand from 2004 to 2008 was taken from the report of the Thai Meteorological Department (TMD) and Hydro and Agro Informatics Institute (HAII). The meteorological data included temperature, atmospheric pressure, specific humidity, windspeed, and rainfall. The meteorological data were taken from monitoring stations near the areas of disease outbreaks within a radius of about 100 kilometers.

2.1.3 The procedures for data preparation concerning meteorology consisted of first, the one-day meteorological record of an onset date, which was the date before the AIV outbreak was officially announced in Thailand, which corresponded to the OIE and DLD criteria since AIV was found to be a symptom of sick animals, and second, the delta $(\Delta X)$ representing the maximum gradient of meteorological parameters in 24 hours (Table 1). The following description presents the meaning of each variable in the equations below: (1) $X i$ was the maximum of the meteorological data over 24 hours, and $X j$ was the minimum of the meteorological data over 24 hours. The specific humidity was calculated by equation (2). $Q$ was the specific humidity $(\mathrm{g} / \mathrm{kg}), p$ was the total atmospheric pressure $(\mathrm{mb}), e$ was the vapor pressure $(\mathrm{mb})$ calculated by equation $(3), f$ was the relative humidity in percent, and $e_{m}$ was the saturation vapor pressure (George, 1965).

$$
\begin{aligned}
& \Delta X i=X i_{\text {maximum }}-X j_{\text {minimum }} \\
& Q=\frac{622 e}{p} \\
& f=\frac{e}{e_{m}} x 100
\end{aligned}
$$

2.1.4 The variance was defined on one day before the OIE and DLD officially announced the onset of AIV outbreak in Thailand, because H5N1 disease takes 24 hours for incubation in poultry.

2.1.5 The data collection of environmental factors consisted of physical data such as the distance between the surrounding villages and the onset AIV village, distance of a stream to the onset AIV village, distance of a road to the onset AIV village, distance of the last outbreak to the onset AIV village, and the density of villages in a radius of five kilometers.

2.1.6 The selection criteria for the study area were based on information about the outbreaks of H5N1 from DLD. DLD determined the circle of the epidemic, which was taken as the period from the first report on the outbreak until the surveillance ended and there was no further report on the outbreak. If there was a new report on the outbreak, the circle of epidemic would start again.

\subsection{Statistic Analysis}

2.2.1 The data $\Delta X_{\mathrm{i}}$ was analyzed by descriptive statistics and multiple regressions.

2.2.2 The data was obtained through the process of multiple regression analysis. The variable was $k\left(X_{1}, X_{2}\right.$, $X_{3}, \ldots, X_{\mathrm{k}}$ ) correlated with the dependent variable $Y$ by the multiple regression equations. Equation (4) was used for checking the criteria of variables before analysis, which consisted of steps: 1) dependent variable must be quantitative whereas independent variable can be both quantitative and categorical; 2) all independent variables $\left(X_{1}, X_{2}, ., X_{\mathrm{k}}\right)$ can be separated from one another; 3$)$ average of the errors equals zero $\left.(\mathrm{e}=0) ; 4\right)$ the margin of error has normal distribution; and 5) the variance of e, $\sigma^{2}$, is constant for all values of $X$ (Kalaya, 2008). If $Y$ is high and suggests that the environment affects the limiting factors of AIV infection, this indicates that the environment is conducive to survival of the virus.

$$
Y=\beta_{0}+\beta_{1} X_{1}+\beta_{2} X_{2} \ldots+\beta_{\mathrm{k}} X_{\mathrm{k}}
$$

where 


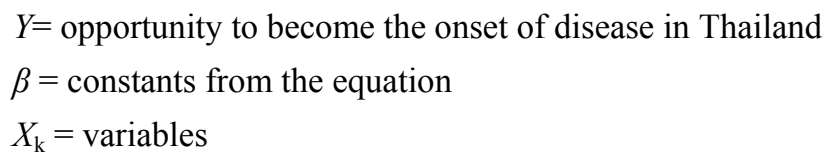

\section{Results and Discussions}

The phenomena of AIV onsets in Thailand happened seven times from 2004 to 2008 (Fig. 1). In 2004, AIV was found in a layer chicken farm $(a, b)$; in 2005 it was found in a partridge farm $(c)$; in 2006 it was found in backyard chicken in one village $(d)$; in 2007 it was found in a layer duck farm and in backyard chicken $(e)$ and in 2008 it was also found in backyard chicken $(f, g)$. The study areas for this research covered seven provinces including Suphan Buri, Phichit, Phitsanulok, Phra Nakhon Si Ayutthaya, Sukhothai, Nakhon Sawan, and Uthai Thani (Fig. 2). These areas reported repeated outbreaks, especially in some provinces such as Suphan Buri, Phitsanulok, and Phichit, which reported outbreaks more than twice.

According to descriptive statistics, the ratio of the occurrence of AIV was 1,098,579 ( $\pm 1,097,818)$, Delta temperature was $8.3( \pm 2.6)$ celsius $\left({ }^{\circ} \mathrm{C}\right)$, Delta atmospheric pressure was $5.8( \pm 2.8)$ hectopascals $(\mathrm{hPa})$, Delta specific humidity was $2.5( \pm 0.5)$ grams per kilogram $(\mathrm{g} / \mathrm{kg})$, Delta wind speed was $0.8( \pm 0.2)$ meters per second $(\mathrm{m} / \mathrm{s})$, and average rainfall within 24 hours was $1.2( \pm 3.4)$ millimeter $(\mathrm{mm}$.$) . Besides the above, physical$ environmental factors showed that the number of villages around the onset village in a radius of five kilometers was $15.6( \pm 8.36)$ villages/five kilometer; the density of villages in a radius of five kilometers was $-0.8( \pm 0.2)$ village/density of village in onset area; the distance between the last point of the outbreak and the onset of outbreak was $30.6( \pm 17.3)$ kilometers $(\mathrm{km}$.); the distance of water from the onset area was $2.6( \pm 0.7) \mathrm{km}$; and the distance of a road from the onset area was $1.8( \pm 2.5) \mathrm{km}$.

The analysis of multiple regression found that there was a relationship between the environmental factors and the onsets of AIV outbreak such as delta temperature ( $p$-value $=0.02)$, delta wind speed $(p$-value $=0.014)$, the density of villages in a radius of five kilometers ( $\mathrm{p}$-value $=0.015)$, distance of the last outbreak to the onset AIV village ( $p$-value $=0.045$ ), and the distance of the stream to the onset AIV village ( $p$-value $=0.051)$. Factors, according to the patterns of relationship, can be divided into 2 groups. Firstly, the dynamic factors such as delta temperature and delta wind speed, and secondly, the static factors such as the density of villages in a radius of five kilometers, and the distance of the last outbreak to the onset AIV village. Furthermore, the results showed that if the possibility of getting symptoms for the onset of disease $(\mathrm{Y})$ increases, it will contribute to the increase in the values of four environmental factors. In the other words, if environmental factors are disappear, the possibility of getting illness will not occur.

Opportunity for the onset to occur required 4 factors. These factors influence one another, thus the onset could not happen if the composition was incomplete. For example, if the difference of daily temperature changes a great deal, the difference of wind speed would increase as well. This is because a higher temperature will cause a hot air mass, causing the phenomenon called convection. Then, the cold air mass will replace the rising hot air mass, which is called the advection. If the rising of the hot air mass happens quickly, the cold air mass will also move in quickly to replace it, and this causes the wind. Furthermore, the difference in temperature and humidity causes changes in air mass making a cold front with cold air displacing warm air, and warm front with warm air displacing cool air. From the meteorological data for Thailand in 2004-2008, it was found that there was more water vapor three days before the onset date because four to seven days before the onset date there may have been rain. This phenomenon caused changes in weather conditions, making high humidity before it rained. Therefore, once it was raining, the animals' health would be affected as they could not adjust to the changes of weather and thus their health was weakened. The environmental factors resulted in healthy animals being susceptible, rather than being an issue of distribution of the infection or pollutants in the air.

Wind speed, besides the relation with temperature, is also related to geology. In other words, the onset areas were agricultural areas such as paddy fields, which were an obstruction to the influence of wind speed. According to the principle of Surface Roughness, the wind speed in agricultural areas, for example, does not change because there is less obstruction than in the city.

The density of villages in a radius of five kilometers and the distance from the last outbreak to the onset AIV village are relevant. Where there is a higher density of villages, the community is larger. This makes it easy for extensive transmission of AIV carriers inside the community. Moreover, if the distance between the last onset and the community area is small, it is easy for the carriers, which are infected from the last onset, to incubate the disease and cause the next pandemic circle once there are appropriate meteorological factors. 


\section{References}

Åkesson, S. and Hedenström, A. (2000). Wind selectivity of migratory flight departures in birds. Behavioral Ecology and Sociobiology. 47, 3: 140-144.

Bureau of Disease Control and Veterinary Services. (2005). Guidelines for Avian Influenza Control. Department of Livestock Development. $2^{\text {nd }}$ ed. The Agricultural Co-operative Federation of Thailand, Ltd. Printing. Bangkok Thailand. $61 \mathrm{p}$.

Bureau of Epidemiology. (2004). Epidemiological Surveillance Annual Report 2547. http://203.157.15.4/Annual/ Annual47/ part1/16-\%E4\%A2\% E9\%CB\%C7\%D1\%B4\%B9\%A1.pdf (online) 1 June 2009

Bureau of Epidemiology. (2005). Epidemiological Surveillance Annual Report 2548 [Online] Available: http://203.157.15.4/Annual /Annual48/Part1/16-Avian\%20influenza.doc (online) 1 June 2009

Bureau of Epidemiology. (2009). Surveillance of avian influenza in humans. [Online] Available: http://203.157.15.4/surdata/ai/ dailyreport/y52/r280952.pdf (online) 7 October 2009

Fouchier, R.A., Munster, V., Wallensten, A., Bestebroer, T.M., Herfst, S., Smith, D., Rimmelzwaan, G.F., Olsen, B., Osterhaus, A.D. (2005). Characterization of a novel influenza A virus hemagglutinin subtype (H16) obtained from black-headed gulls. J Virol. 79: 2814-22.

George F. Taylor, G. F. (1965). Elementary Meteorology. $8^{\text {th }}$ ed. New York: Prentice-Hall; Englewood Cliffs, N.J., USA. 366

Kanlaya Vanichbuncha, K. (2008). SPSS for Windows. $11^{\text {th }}$ ed. Bangkok: Chulalongkorn University Press, Thailand. 520

Songserm, T., Sae-Heng, N., Jam-on, R., and Meemak, N. (2005). Abstracts of the OIE/FAO International Conference on Avian Influenza. In survival of highly pathogenic avian influenza H5N1 in different conditions and susceptibility to disinfectants. Page 1-7. Paris; 7-8 April 2005.

Sturm-Ramirez, K. M., and others? (2005). Are ducks contributing to the endemicity of highly pathogenic H5N1 influenza virus in Asia? Journal of Virology. 79, 17 (2005): 11269-11279.

Webster, R.G., Bean, W.J., Gorman, O.T., Chambers, T.M., Kawaoka, Y. (1992). Evolution and ecology of influenza A viruses. Microbiological Reviews. 56, 1: 152-79.

World Health Organization. (2009). Cumulative number of confirmed human cases of avian influenza A/(H5N1) reported to WHO 28th May 2009 [Online] Available: http://www.who.int/csr/disease/avian_influenza/ country/cases_table_2009_05_28/en/index.html (online 1st June 2009)

Zhou, J.Y. et al.? (2006). Characterization of a highly pathogenic H5N1 influenza virus derived from bar-headed geese in China. Journal of General Virology. 87:1823-1833. 
Table 1. Definitions of the variables

\begin{tabular}{ll}
\hline \multicolumn{1}{c}{ Variable } & \multicolumn{1}{c}{ Definition } \\
\hline $\begin{array}{l}\text { Opportunity to become the onset of disease } \\
\text { in Thailand (Y) }\end{array}$ & $\begin{array}{l}\text { the opportunity to become the starting point of AIV } \\
\text { outbreak in Thailand } \\
\text { the number of positive AIV reports from a laboratory during } \\
\text { each time divided by all reports in this time } \\
\text { matio of AIV }\end{array}$ \\
meximum temperature during the day minus minimum \\
temperature during the day (temp $p_{\text {max }}$-temp $p_{\text {min }}$ ) \\
maximum atmospheric pressure during the day minus \\
minimum atmospheric pressure during the day \\
(pres
\end{tabular}




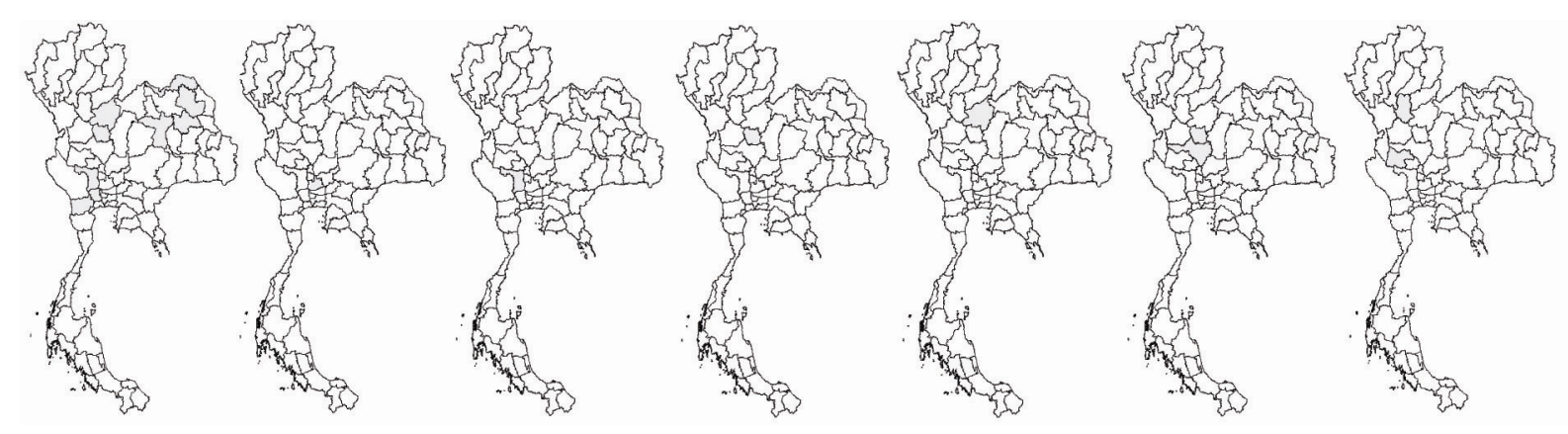

Figure 1. Kickoff areas in Thailand, 2004-2008
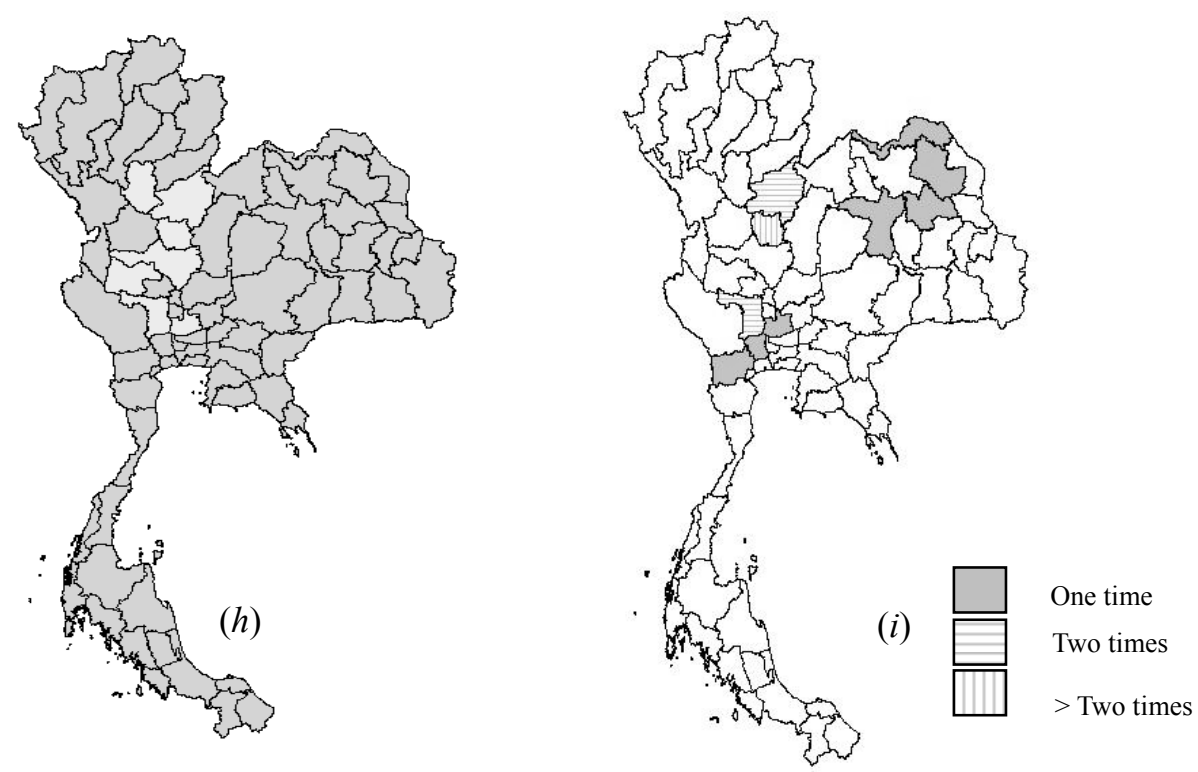

Figure 2. Gray in $(h)$ represents the study areas and colors in $(i)$ represent the repeated outbreak areas 